April - 2004

\title{
Book Review - Reflections on Teaching and Learning in an Online Master Program: A case study
}

Editors: Ulrich Bernath and Eugene Rubin (2003). Reflections on Teaching and Learning in an Online Master Program: A case study. 295 pages, softcover. Oldenburg: Bibliotheks-und Informationssystem der Carl von Ossietzky Universität Oldenburg. ISBN: 381420848 X

Reviewed by: Dianne Conrad, University of New Brunswick, Canada

In his review of a recent publication on leadership strategies in open and distance learning, Don Olcott commended the editors of that publication on their fine contribution, noting that there really is no substitute for experience in helping distance education's practitioners, leaders, and novices learning about open and distance education to acquaint themselves with the theory and practice that define the field. Bernath and Rubin's Reflections on Teaching and Learning in an Online Master Program gives us that rich study in experience as they examine online teaching and learning issues through the lens of the Master of Distance Education program (MDE), offered jointly by the University of Maryland University College and Carl von Ossietzky University of Oldenburg, Germany. This volume is the most recent publication in the Carl von Ossietzky University of Oldenburg's series from the Center for Research in Distance Education, Volume 6 of an intended 10.

The book contains 11 chapters divided into three parts. The first part outlines the basic structure of the program and details the organizational and managerial issues involved in the mounting and delivering of online programs. In the second part of the book, program faculty and visiting scholars draw upon their experiences to examine program issues from their respective positions. The third part gives voice to the program's students. To complete this volume, a series of indexes provide: a name index, an index of useful acronyms, and a highly detailed subject index.

Strategically, contributors' names, many of which are highly recognizable, are placed near the front of the book. As such, it falls upon the shoulders of these contributors to broad-brush the program and capture, theoretically and conceptually, the fundamental issues that inform the MDE case study. In their opening chapter, editors Bernath and Rubin outline the history of their joint program, tracing its political roots and development from its 1997 genesis in the experimental "Virtual Seminar for University Faculty and Administrators 'Professional Development in Distance Education"” program. We know from many recent studies in online education, that the details constituting a particular program's model of delivery are key to understanding that program's rationale, protocols, and resultant success. To this end, Bernath and Rubin describe the MDE by providing its early developmental history, course descriptions, lists of faculty, students' comments, and ample assessment information, including a detailed description of their assessment instruction and students' ratings. An appendix containing excerpts from their initial proposal will be of interest to those who may be contemplating putting forward similar proposals. 
Otto Peters' chapter on moderating a virtual seminar powerfully combines a career's worth of theory-based practice with his hands-on experience teaching in the MDE program. The case study format melds the immediacy of research's raw data with thoughtful interpretation: a bonanza of insight into the nuts-and-bolts of online teaching and learning. Peters' chapter is anchored by first-person narrative from the learners' perspective, the replication of email messages, and a section where he shares his personal techniques for communicating with seminar learners.

The writing here is forthright and practical, demonstrating an accessibility not always found in research papers. A full page lists the advantages of virtual seminars, as offered by the learners (who are identified by their real names!). This chapter's lengthy appendix also contains valuable information: copies of correspondence used to set up the seminar and copies of evaluation instruments. The format, unfortunately, remains clumsy and uncomfortable to read, as is the case throughout the volume.

Thomas Hülsmann's chapter (there are no chapter numbers!) addresses the intriguingly entitled concept of "texts that talk back" - a look at the potential for academic discourse in asynchronous fora. I personally found this chapter to be one of the most information-laden pieces of work I have ever encountered in our field. Thorough, detailed, anecdotal, research-based, Hülsmann plays ample quantitative data against references to Plato, Hegel, and McLuhan to create a deeply intelligent discussion on the nature of asynchronicity. Using the concepts of richness and noise, he captures the dichotomous nature of this medium wherein successful learners must "pursue their own learning agenda and, at times, link it to that of their peers" (p. 90). Section 4 of this chapter features an extremely detailed, graphic, and statistical 12-page "experiential" description of an MDE course. Too much information? Perhaps. I gave my students permission to "read by" it. A very eclectic and lengthy bibliography is followed by another statistically-intensive sevenpage appendix.

By comparison, Beaudoin's short chapter on "invisible" online learners was much more reminiscent of a journal publication. Explained by the author as representing only the results of a preliminary case study, Beaudoin nonetheless furthers our understanding of the nature of interaction and learning success through an articulate presentation of research-based findings.

The remainder of the second part of the volume addresses topics of support and costs. The title of Holmberg's short chapter suggests at first that it will discuss the issue of support, but it provides instead a brief overview of computer use in education. While slightly out of character with the intensive case-study orientations of other chapters, its conceptual discussion of the role of the computer in distance education is remains useful. Holmberg reminds us that the computer "does not represent the essence of distance education, which seems occasionally to be assumed. ..”

Those of us who have been responsible for constructing or delivering distance education programs appreciate the need for institutional support and infrastructure. In the chapter entitled, "Support Services for Online Faculty," the authors - administrators of, and teachers within, the MDE program - explore the key issues underlying the establishment of a supportive institutional culture. As befits a case study and as is typical of this volume, their discussion is detailed and information-rich. Its qualitative nature and structure renders this chapter more "reader-friendly" than some of the others in this volume. It is nicely referenced, drawing on a balance of internationally known sources and local documents. 
Hülsmann's second contribution to this work addresses the "dearth of cost data" that is made available on institutional projects such as this one. He does so in a very long 57-page chapter (It might be noted here that this chapter surpasses the combined length of the four student-authored chapters.) That said, Hülsmann's chapter contains, again, minutely detailed financial planning and analytical data as well as, surprisingly, methodological references that use Lincoln and Guba to explain the chapter's Geertz-like "thick description" and to differentiate between the concepts of generalizability and transferability. "Costs without camouflage" purports to strip away the cloak of secrecy that so often prevents the disclosure of distance education's true costs. To that end, Hülsmann's chapter includes answers to questions that "are asked again and again when it comes to online learning" and very detailed decompositions of related budgets and costs. The complexity of both building distance education programs and costing them, however, taxes the author's attempts to present a transferable understanding of his program's financial landscape. This is a difficult chapter that reminds us, once again, of the idiosyncratic existence of most innovative programming.

The concluding third section of Bernath and Rubin's monograph comprises four selections from participants in the MDE program. Collectively, the four chapters number only 49 pages: as short, topical, academically well-referenced papers they work well. While all offer insights on aspects of the MDE's mandate and implementation, only Fox's chapter presents a learner's personal account of his experience in the program. As such, it is a valuable commentary, raising questions where necessary, and providing insight into, among other things, areas of dissention among learners. Like the other chapters in this final section, Fox's work has been cross-referenced to other related topics discussed in the monograph.

If we can indeed learn from the experiences of others, then the MDE experience, well documented within this case study, is a valuable teaching tool. If you were to seek out this volume, however, and find it on the shelf, its poor first impression may well discourage you from looking past its exterior and discovering the value within its pages. Its appearance and style are decidedly un-bookish, resembling instead a legal journal from the outside and a technical report on the inside. The first page of each chapter is structured as a table of contents with an overwhelming amount of pagination detail. The chapters themselves have no numbers, making it difficult to refer to a particular piece, except as, for example, "the Peters chapter, on page 51." Valuable appendices to chapters are produced entirely in italics, and the labeling of figures and tables is often cramped and confusing. Charts and tables, laden with quantitative data, are squeezed in small fonts into busy pages. The awkward use of bullets, white space, indentations, quotation marks, and fonts often contributed to a sense of visual confusion for this reader.

In spite of that, this is a rich source of practical and applied knowledge that unflinchingly tackles the difficult and thorny questions that go hand-in-hand with innovation program implementation. Don't judge this book by its cover!
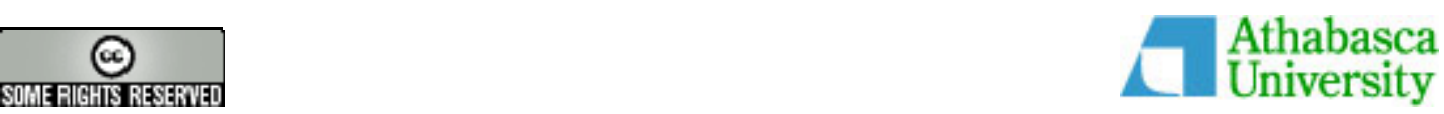